\title{
An Effective Three-Dimensional Layout of Actuation Body Force for Separation Control
}

\author{
Ittetsu Kaneda, ${ }^{1}$ Satoshi Sekimoto, ${ }^{1}$ Taku Nonomura, ${ }^{2}$ Kengo Asada, ${ }^{1}$ \\ Akira Oyama, ${ }^{2}$ and Kozo Fujii' \\ ${ }^{1}$ Department of Aeronautics and Astronautics, University of Tokyo, Bunkyo, Tokyo 113-8656, Japan \\ ${ }^{2}$ Department of Space Transportation Engineering, Institute of Space and Astronautical Science, Japan Aerospace Exploration Agency, \\ Sagamihara, Kanagawa 252-5210, Japan
}

Correspondence should be addressed to Taku Nonomura, nonomura@flab.isas.jaxa.jp

Received 17 August 2011; Revised 5 December 2011; Accepted 24 January 2012

Academic Editor: Siao Chung Luo

Copyright (c) 2012 Ittetsu Kaneda et al. This is an open access article distributed under the Creative Commons Attribution License, which permits unrestricted use, distribution, and reproduction in any medium, provided the original work is properly cited.

\begin{abstract}
We conducted large eddy simulations of the control of separated flow over an airfoil using body forces and discuss the role of a three-dimensional vortex structure in separation control. Two types of cases are examined: (1) the body force is distributed in a spanwise uniform layout and (2) the body force is distributed in a spanwise intermittent layout, with three-dimensional vortices being expected to be generated in the latter cases. The flow fields in the latter cases have a shorter separation bubble than those in the former cases although the total momentum of the body force in the latter cases is the same as or half of the former cases. In the flow fields of the latter type, the three-dimensional vortices, which are not observed in the former cases, are generated by the body force downstream of the body force distributed. Thus, three-dimensional vortices are considered to be effective in controlling the separated flow.
\end{abstract}

\section{Introduction}

Recently, the dielectric barrier discharge (DBD) plasma actuator [1] (hereafter, "plasma actuator") — a small active flow control device-has been receiving a lot of attention because it is superior to conventional devices with respect to reactivity, a comparatively simple structure, and smaller energy consumption. The plasma actuator can probably find applications in unmanned air vehicles (UAVs) [2] and turbine blades $[3,4]$.

In this paper, we investigate the potential applications of a plasma actuator in separation control. Figure 1 shows a schematic diagram of a plasma actuator. As shown, a plasma actuator consists of two electrodes and a dielectric. A plasma actuator generates plasma by means of dielectric barrier discharge in the area between the exposed electrode and the dielectric when a high alternating current $(\mathrm{AC})$ voltage is applied to the electrodes, inducing flow.

Many experiments and numerical simulations are conducted in this study, and their results show the applicability of a plasma actuator for use in separation control. In particular, a lot of studies are conducted on the input AC parameters (e.g., voltage, frequency, and waveform) [5, 6]. With respect to the waveform shown in Figure 2, it has been found that using an unsteady input voltage, or "burst wave," gives a better separation control capability $[5,7,8]$. The nondimensional burst wave frequency $F^{+}$is set between 1 and 10 in these studies.

Research conducted to clarify the mechanism of separation control is mainly performed by means of numerical simulations. Asada and Fujii investigated the flow field around an airfoil controlled by the plasma actuator, that was operated by both a burst wave and a normal AC wave to clarify the mechanism of separation control [9]. This research showed that the three-dimensional turbulent vortices generated after the breakdown of spanwise twodimensional vortices promote flow mixing, and greatly contribute to separation control. This result implies that the generation of three-dimensional turbulent vortices is the key to separation control, which leads to the supposition that the 


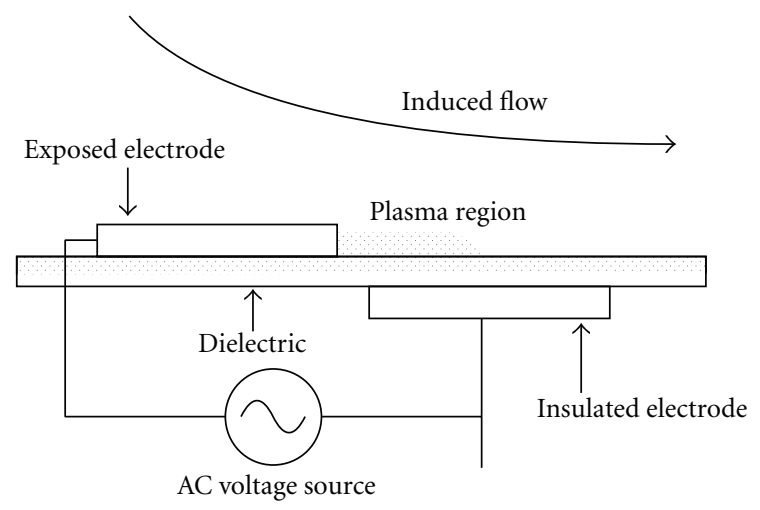

FIGURE 1: Configuration of plasma actuator.

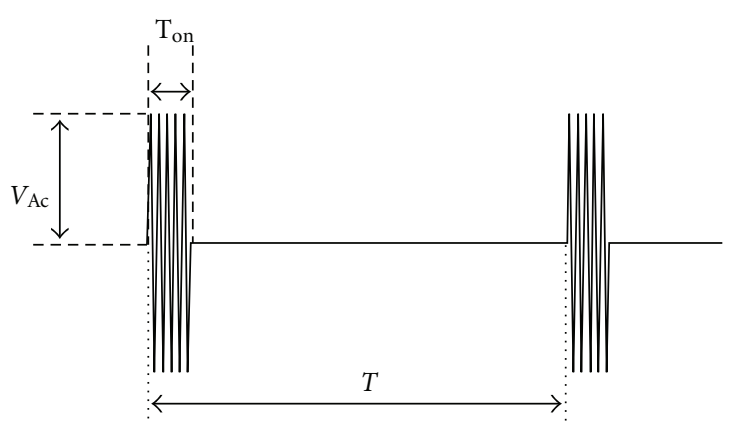

Figure 2: Burst wave image.

intentional generation of three-dimensional vortices would be an effective method of separation control.

With regard to the research in which three-dimensional turbulent vortices are generated intentionally and the effect of separation control is investigated, a research has been conducted where a spanwise nonuniform body force of a plasma actuator is distributed. Poggie et al. performed an experiment in which a plasma actuator with a finger configuration is applied to an airfoil [10]. In their research, the main purpose was to clarify whether a hot-film sensor could determine a reattached point, and thus a plasma actuator with a finger configuration was not compared with the plasma actuator of a spanwise uniform configuration. In addition, the relation between three-dimensional vortices and the effect of separation control was not remarked upon, although it was remarked that the plasma actuator of a finger configuration affects the separation control. Rizzetta and Visbal conducted a numerical simulation in which a plasma actuator generates three-dimensional vortices intentionally [11]. In their research, two cases were calculated using several force models and grids. In the first case, the body force of a plasma actuator is distributed spanwise uniformly on an airfoil. In the second case, the body force of a plasma actuator is distributed spanwise intermittently. The results showed that the latter case is more effective in terms of turbulent transition than the former case. However, the relation between three-dimensional vortices and separation control was not remarked upon in details.
Thus, our research aims to understand the phenomenon regarding the effects of three-dimensional turbulent vortices on separation control and to figure out the more effective configuration. In order to generate three-dimensional turbulent vortices intentionally, body force is distributed spanwise intermittently. This research employs large-eddy simulation (LES), which is considered to be adequate to allow an understanding of an unsteady flow field. Two types of body force distributions are considered: (1) a spanwise uniform layout and (2) a spanwise intermittent layout. In the first type, three cases with body forces of different momentum are examined. In the second type, three cases with different intermittent layout distances, but under the same body force, are examined.

\section{Problem Settings}

2.1. Flow Conditions. The flow conditions, used in these experiments, are the same as those used in previous computational and experimental studies by the authors $[9,12]$. The freestream Mach number is set to 0.2 as the compressibility of fluid is almost negligible. The Reynolds number, on the basis of the chord length, is set to 63,000 . The specific heat ratio and the Prandtl number are set to 1.4 and 0.72 , respectively. An NACA 0015 airfoil is used with the angle of attack set to $14 \mathrm{deg}$. The same body force model is used in all the experiments and is installed on the airfoil surface at $5 \%$ of the chord length from the leading edge.

2.2. Governing Equations. Three-dimensional Navier-Stokes equations, nondimensionalized by the freestream density, freestream velocity, and the chord length of the airfoil, are employed as the governing equations in this study. In the nondimensional form, the governing equations are represented as follows:

$$
\begin{gathered}
\frac{\partial \rho}{\partial t}+\frac{\partial \rho u_{k}}{\partial x_{k}}=0 \\
\frac{\partial \rho u_{i}}{\partial t}+\frac{\partial\left(\rho u_{i} u_{k}+p \delta_{i k}\right)}{\partial x_{k}}=\frac{1}{\operatorname{Re}} \frac{\partial \tau_{i k}}{\partial x_{k}}+D_{c} S_{i}(x, y, z, t), \\
\frac{\partial e}{\partial t}+\frac{\partial\left((e+p) u_{k}\right)}{\partial x_{k}} \\
=\frac{1}{\operatorname{Re}} \frac{\partial u_{l} \tau_{k l}}{\partial x_{k}}+\frac{1}{(\gamma-1) \operatorname{Pr} \operatorname{Re} M_{\infty}^{2}} \frac{\partial q_{k}}{\partial x_{k}}+D_{c} S_{k} u_{k}, \\
S_{i}=Q_{c} E_{i},
\end{gathered}
$$

where $x_{i}, u_{i}, q_{i}, \rho, p, e, \tau_{i j}, \delta_{i j}$, and $t$ denote the nondimensional forms of position vector, velocity vector, heat flux vector, density, pressure, energy per unit volume, stress tensor, Kronecker delta, and time, respectively. Nondimensional parameters Re, $M$, and Pr denote the Reynolds number, Mach number, and Prandtl number, respectively, and are defined as follows:

$$
\operatorname{Re}=\frac{\rho_{\infty} u_{\infty} c}{\mu_{\infty}}, \quad M_{\infty}=\frac{u_{\infty}}{a_{\infty}}, \quad \operatorname{Pr}=\frac{\mu_{\infty} c_{p}}{k_{\infty}},
$$


where $\mu, c, a, c_{p}$, and $k$ denote viscosity, chord length, sound speed, constant pressure specific heat, and heat conduction coefficient, respectively. Here, the subscript $\infty$ denotes quantity in a freesteam condition. In (2) and (3), the last terms on the right-hand side represent the body force and the power added to the unit volume by the body force, respectively. Details of the body forces are described in the next subsection.

2.3. Modeling of the Plasma Actuator. In the previous subsection, it was stated that the body force is modeled with $D_{c} Q_{c} E_{i}\left(=D_{c} S_{i}\right)$ and $D_{c} Q_{c} u_{k} E_{k}\left(=D_{c} u_{k} S_{k}\right)$ in the NavierStokes equations. Here, $S_{i}$ denotes the nondimensional body force vector, $Q_{c}$ denotes the electric charge, and $E_{i}$ denotes the electric-field vector. The nondimensional plasma scale parameter $\left(D_{c}\right)$ is defined as

$$
D_{c}=\frac{Q_{c, \text { ref }} E_{\mathrm{ref}} c}{\rho_{\infty} u_{\infty}^{2}},
$$

where the subscript ref denotes the reference value. Here, we set $Q_{c \text {,ref }}$ and $E_{\text {ref }}$ as maximum values of $Q_{c}$ and $E$ in the simulation results for the Suzen model [13]. The nondimensional plasma scale parameter $\left(D_{c}\right)$, used in this study, is similar to previous studies. We could obtain $E_{\text {ref }}$ from the input voltage by setting the $D_{c}$ value, on the basis of experimental settings, but could not determine $Q_{c, \text { ref. }}$ Thus, we conducted an a priori test to examine the dependency of the maximum induced velocity while varying $D_{c} ; D_{c}$ is set to ensure that the ratio of the maximum induced velocity and the freestream velocity is a realistic value. Here, $D_{c}=8$ corresponds to actuation in which the maximum induced velocity becomes approximately equal to the freesteam velocity.

The distribution of $S_{i}$ is determined by a Suzen model [13]. Figure 3 shows the body force vector distribution of the Suzen model. The contoured surface denotes the body force magnitude, and the yellow vectors denote the body force direction. The two white lines represent the electrodes. This model has two characteristic body forces. The first is the force vertical to the airfoil surface, and the second is the force along the airfoil surface. Figure 4 shows the body force magnitude distribution for the computation used in this paper. The dimension of the model region is 0.015 (chord direction) $\times 0.01$ (vertical to the chord direction). The center of the model region, in the direction vertical to the chord direction, is on the body surface. There is zero body force in the span direction. This body force model is rotated around the point indicated by the red arrow and is installed on the airfoil surface at $5 \%$ of the chord length from the leading edge.

2.4. Operating Conditions of Body Force. There are two types of layouts: "standard layout" and "intermittent layout" where cases of standard layout are the cases in which the body force is distributed in a spanwise-uniform layout, and cases of intermittent layout are the cases in which the body force is distributed in spanwise-intermittent layout, whereas the "on" and "off" width is the same. The flow field is compared to the

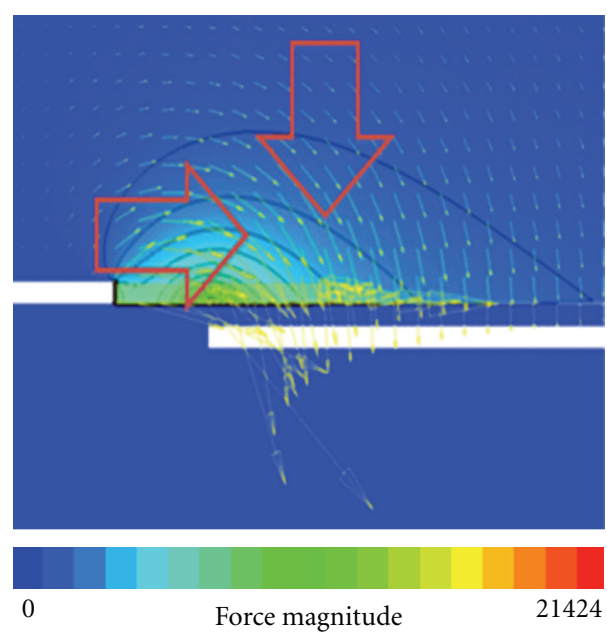

FIgURE 3: Force image of Suzen model.

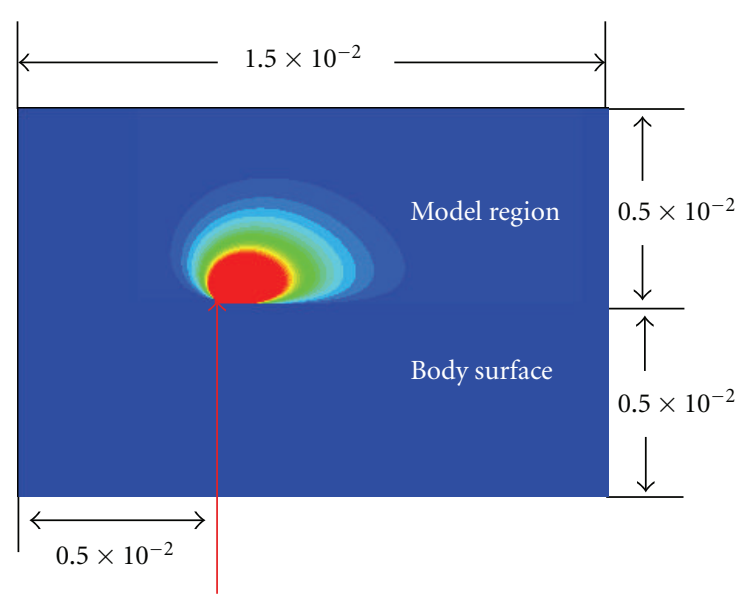

Figure 4: Force distribution of Suzen model.

flow field in the absence of body force, called "DBD-off." An unsteady body force fluctuation is formulated as follows:

$$
S(x, y, z, t)=S_{\text {suzen }}(x, y, z) \sin ^{2}\left(2 \pi F_{\text {base }} t\right),
$$

where $F_{\text {base }}=f_{\text {base }} c / U_{\infty}$ is the nondimensional base frequency for the $\mathrm{AC}$ of the input voltage. Here, $f_{\text {base }}$ is the base frequency for the $\mathrm{AC}$ of the input voltage. In addition, we maintain the nondimensional base frequency $\left(F_{\text {base }}\right)$ at 60 , which is much higher for flow fluctuation and appears to be in steady actuation. Although the use of burst mode actuation with $F^{+} \sim O$ (1) with a spatially intermittent layout appears promising, only a normal actuation is considered for simplicity in this study. Moreover, the plasma actuator fluctuation is modeled using a square of sine function because the force generated by the plasma actuator is termed as a push-push type in one cycle which is similar to the square of sine function, and not push-pull type which is similar to the sine function [14]. $D_{c}$ is set to 0 where the body force is not added in the intermittent cases. Spanwise averaged $D_{c}\left(D_{c \text {,ave }}\right)$ is used as the parameter for denoting the strength of the body force, where $D_{c}$ in the region where the 
TABle 1: Computational cases.

\begin{tabular}{lcccc}
\hline Case name & Body force layout & $D_{\text {c,ave }}$ & $F_{\text {base }}$ & Intermittent distance (wall unit) \\
\hline DBD-off & N/A & 0 & N/A & N/A \\
Std-8 & Standard layout & 8 & 60 & N/A \\
Std-16 & Standard layout & 16 & 60 & N/A \\
Imt-0.006 & Intermittent layout & 8 & 60 & $0.006 c(37.7)$ \\
Imt-0.018 & Intermittent layout & 8 & 60 & $0.018 c(93.9)$ \\
Imt-0.045 & Intermittent layout & 8 & 60 & $0.045 c(237.7)$ \\
\hline
\end{tabular}

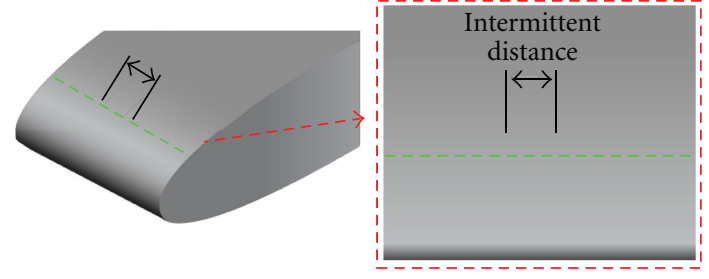

FIGURE 5: Intermittent layout of body force.

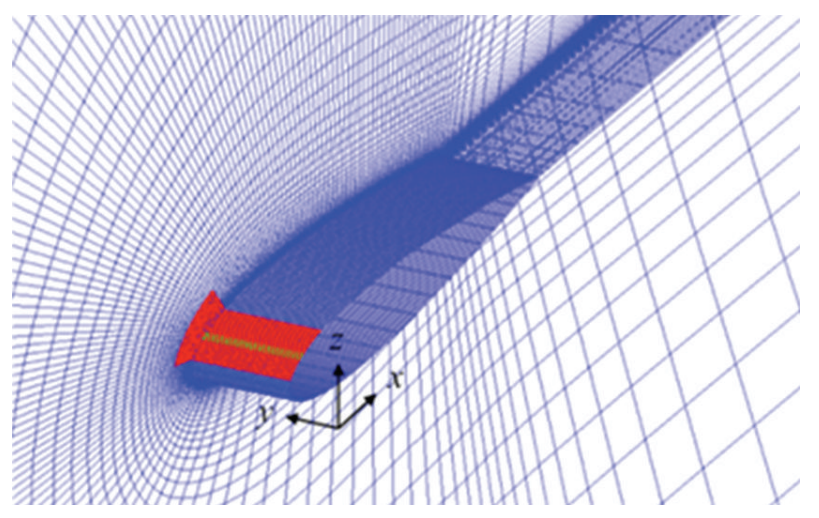

FIgURE 6: Computational grids (air view).

body force does not work is estimated as zero. $D_{c \text {,ave }}=8$ or 16 in the standard cases and $D_{c \text {,ave }}=8$ in the intermittent cases are chosen as realistic $D_{c \text {,ave }}$ values. However, in the intermittent cases, $D_{c}$ is approximately twice the value in the region where the body force works to obtain $D_{c \text {,ave }}$ having the same value. Table 1 shows the computational cases in this research. In this table, DBD-off is the case in which the body force is zero. In intermittent cases, the body force is distributed spanwise intermittently, as shown in Figure 5. The body force distribution is uniformly intermittent, and this distance is shown as intermittent distance in Table 1. We set the same length for the forcing part and the nonforcing part, and the intermittent distance corresponds to each part in both cases.

\section{Computational Approach}

3.1. Numerical Method. In this study, LANS3D $[15,16]$, a fluid analysis solver developed at the ISAS/JAXA, is employed for the calculations. Three-dimensional compressible Navier-Stokes equations are employed as the governing

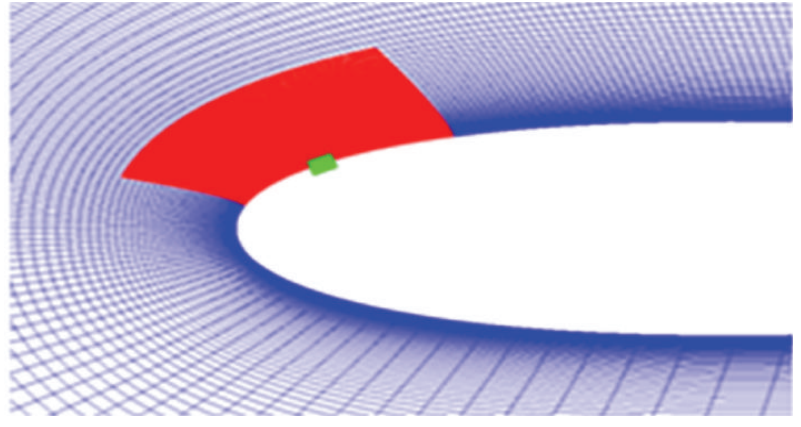

FIGURE 7: Computational grids near the leading edge.

equations. These equations are solved in the generalized curvilinear coordinates $(\xi-\eta-\zeta)$. As the velocity induced by the body force is very small and unsteady, a highresolution unsteady computational method is required. Thus, the spatial derivatives of the convective and viscous terms, metrics, and Jacobian are evaluated by a sixthorder compact difference scheme [17]. Near the boundary, second-order explicit difference schemes are used. Tenthorder filtering $[18,19]$ is used with a filtering coefficient of 0.47 . For time integration, lower-upper symmetric alternating direction implicit and symmetric Gauss-Seidel (ADISGS) [20] methods are used. To ensure time accuracy, a backward second-order difference formula is used for time integration, and three subiterations [21] are adopted. The computational time step is $4 \times 10^{-5}$ in nondimensional time to obtain a maximum Courant-Friedrichs-Levy (CFL) number of approximately 1.7. In a standard LES approach, additional stress and heat flux terms are appended, but in an implicit ILES [22] approach, they are not appended. In this research, ILES is employed, and a high-order, low-pass filter selectively damps only poorly resolved high-frequency waves. This filtering regularization procedure provides an attractive method for the use of standard sub-grid-scale (SGS) models. At the outflow boundary, all variables are extrapolated from one point in front of the outflow boundary. For the airfoil surface, no-slip conditions are adopted. A periodic boundary condition is applied to the boundaries in the spanwise direction.

3.2. Computational Grids. The zonal method [22] is employed to treat the region of small body force. Figures 6 and 7 show the computational grids, where a body force 
model is applied at $5 \%$ of the chord length from the leading edge. The grids for the ILES consist of two parts: a blue airfoil grid (zone 1) and a fine red grid (zone 2). The computation procedure consists of the following three steps. Firstly, the body force of the Suzen model is calculated on the green grid corresponding to the body force model region. Then, the body force is mapped to the zone 2 grid from the green grid. Then, we solve (1), (2), and (3) in zone 1 and zone 2, and interpolate the physical values from each other. Here, zone 1 is a C-type grid, and the length from the airfoil surface to the exterior boundary is 20 times the chord length. The length of the computational region in the span direction $(y$ direction) is $0.18 \mathrm{c}$. In this span length, only two periods are included for the widest intermittent case, but it appears to be sufficient for qualitative discussion. We also conducted a simulation with a span region that was twice as wide, and confirmed that the qualitative characteristics do not change. Table 2 shows the grid points of each zone where zones 1 and 2 have approximately 4,300,000 and 6,100,000 points, respectively. Thus, the total number of the grid points is $10,400,000$. The minimum grid size in the direction normal to the airfoil surface is $0.00012 c$.

3.3. Validity of Computation. Asada and Fujii compared the $C_{p}$ distributions of computation results with those of experimental results in order to validate the computational method, and these results agreed with the experimental result [9]. In this research, the same computational code is adopted, and it is reliable to enable a comparison of the qualitative difference in separation control of the body force.

\section{Results and Discussions}

4.1. Time-Averaged Flow Fields. In this section, the flow fields are averaged in time and spanwise directions. In the intermittent layout cases, both flow fields averaged in the region where the body force is distributed and those in the region where the body force is not distributed are similar to the flow fields averaged in the entire spanwise region. Thus, in this research, the average in the entire spanwise region is adopted for the intermittent layout case. Figure 8 shows the distributions of averaged chordwise velocity and averaged stream lines at $\alpha=14 \mathrm{deg}$. As shown in Figure 8(a), the flow separates at the leading edge in the DBD-off case. In the Std- 8 and Std-16 cases shown in Figures 8(b) and 8(c), respectively, there is no clear effect of separation control, although the angle of the separated shear layer decreases compared to that of the DBD-off case. In the intermittent layout cases shown in Figures $8(\mathrm{~d}), 8(\mathrm{e})$, and $8(\mathrm{f})$, the flow separation at the leading edge is suppressed. This is because the local $D_{c}$ value in intermittent layout cases is higher than the $D_{c}$ value in the standard layout cases, imposed in order to maintain the spanwise averaged $D_{c}$. However, there is another reason in addition to the effect of the $D_{c}$ value, because the intermittent layout cases show a higher effect of separation control than the Std-16 case, which has the same local $D_{c}$. Figure 9 shows the $C_{p}$ distributions on the airfoil surface in all cases. The separated region, shown in Figure 8, is also observed in the
TABLE 2: Grid points.

\begin{tabular}{lcccc}
\hline Grid & $\xi$ & $\eta$ & $\zeta$ & Total \\
\hline Zone 1 & 351 & 101 & 121 & $4,289,571$ \\
Zone 2 & 301 & 101 & 201 & $6,110,601$ \\
Model grid & 1201 & 801 & N/A & 962,001 \\
\hline
\end{tabular}

$C_{p}$ distributions. In the DBD-off, Std- 8 , and Std- 16 cases, $C_{p}$ is distributed flatly on the suction surface and is less than zero at the trailing edge. It means that the flow separates from the leading edge and does not reattach. On the other hand, the separated region in the intermittent layout cases, where $C_{p}$ is distributed flatly near the leading edge, is clearly reduced compared with the other cases, and the separated region in the Imt- 0.018 case is the smallest of the intermittent layout cases. In addition, there is a small peak of $C_{p}$ near $5 \%$ of the chord length in cases of added body force. This is because the velocity is induced by the body force. Thus, this peak near $5 \%$ of the cord length is higher as the $D_{c}$ value is higher, and a peak is not observed in the DBD-off case. Furthermore, the peak of $C_{p}$ near the leading edge, in the case with body force, is a higher value than that in the DBD-off case. This is because the attached flow is accelerated by the body force.

Figure 10 shows the $C_{f}$ distributions on the suction surface in all cases. As shown in Figure 10, the flow in the intermittent layout cases reattaches, and the flow in the standard layout cases does not. In particular, in the Imt- 0.018 case, the separated region is very small, and the flow reattaches after separation-similar to the discussion above. Figure 11 shows $-u^{\prime} w^{\prime}$ corresponding to the Reynolds stress distributions. In Figure 11, the high $-u^{\prime} w^{\prime}$ region is distributed in the region where two-dimensional vortices are generated from the separated shear layer. In the Imt- 0.018 and Imt -0.045 cases, the Reynolds stress is lesser than that in the other cases. In addition, the Reynolds stress is distributed in a minute region for a smaller separated flow region. This is because the angle of the separated shear layer becomes shallower and the Reynolds stress decreases with control of the separated flow.

Thus, in terms of separation control, intermittent layout cases are more effective than standard layout cases. One of the reasons for the effectiveness of intermittent layout cases is the generation of three-dimensional vortices. Of all the cases, the Imt- 0.018 case has the smallest separation region in intermittent layout cases. In the next subsection, we discuss the relation between the three-dimensional vortices and the effectiveness on the separation control.

4.2. Instantaneous Flow Fields. Figure 12 shows the instantaneous flow fields of the cases investigated in this study. In the left side of each figure, the gray surface shows the airfoil surface, and the iso-surface shows the second invariant of the velocity gradient tensors colored by $x$-vorticity. In the right side of each figure, the colored surface shows the chord directional velocity near the wall. In addition, the background is colored by the chord directional velocity. In the DBD-off case, the shear layer, separated from the 


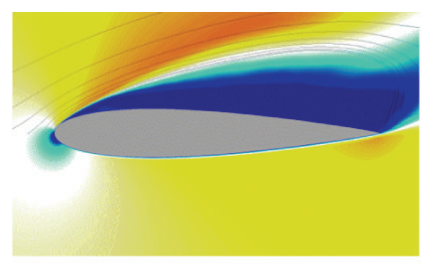

(a) DBD-off case

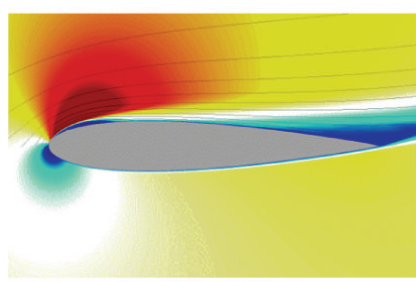

(d) Imt-0.006 case

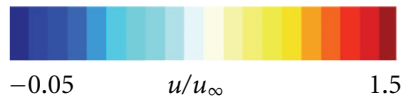

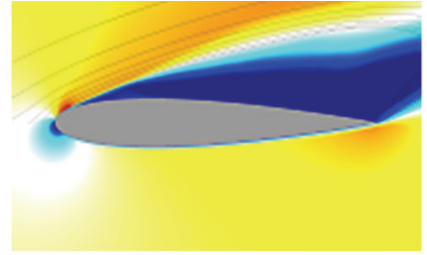

(b) Std-8 case

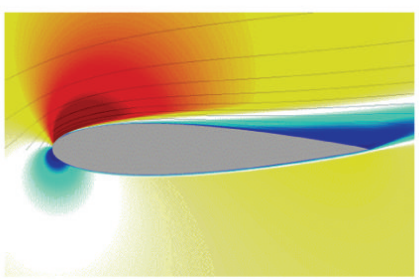

(e) Imt-0.018 case

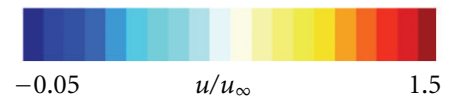

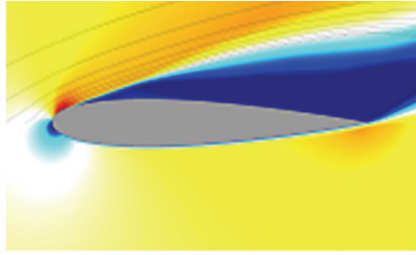

(c) Std-16 case

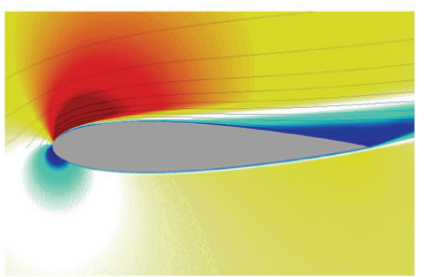

(f) Imt- 0.045 case

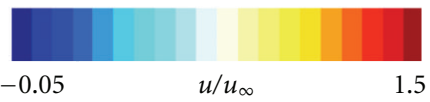

FIGURE 8: Time-averaged and spanwise-averaged chord direction velocity distributions and stream lines.

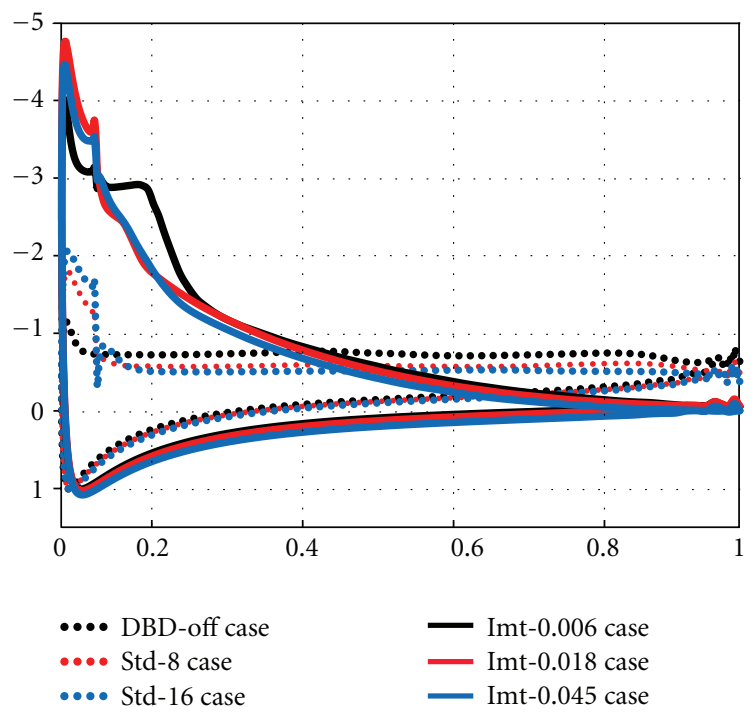

Figure 9: $C_{p}$ distributions.

leading edge, generates two-dimensional vortices on the spanwise axis due to Kelvin-Helmholtz instability. These vortices break down and become complex three-dimensional turbulent vortices heading downstream. In the Std-8 and Std-16 cases, the angle of the shear layer is small, but flow is separated from the leading edge. The two-dimensional vortices are generated from the shear layer and break down into three-dimensional turbulent vortices, as in the DBDoff case. In the Imt- 0.006 case, the two-dimensional vortices break down into three-dimensional turbulent vortices at the region closest to the leading edge. After the transition, the vortices have much smaller structures than those of the standard layout cases. In the Imt-0.018 and Imt-0.045 cases,

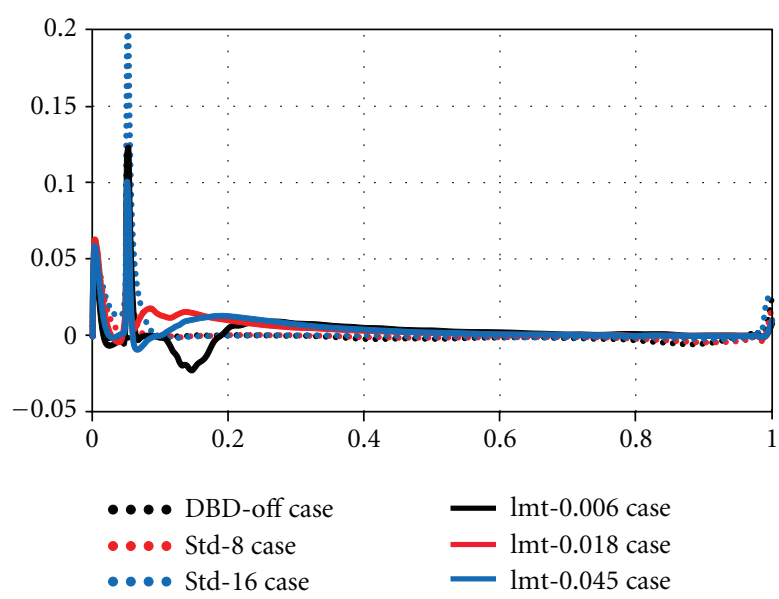

Figure 10: $C_{f}$ distributions.

there are two-dimensional vortices on the area where the body force is applied; however, three-dimensional vortices are behind the 5\% chord length line from the leading edge. In addition, these two-dimensional vortices break down into three-dimensional turbulent vortices, heading downstream. To discuss the transition of vortices, the temporal changes in these vortices are shown in Figures 13, 14, and 15. The colored arrows in the figures indicate the above-mentioned vortices.

In the Imt-0.006 case, as shown in Figure 13, there are no three-dimensional vortices behind the area where the body force is applied, and two-dimensional vortices-such as the vortices in the standard layout case-are generated. However, these vortices are very unstable and break down during downstream transfer more rapidly than those in 


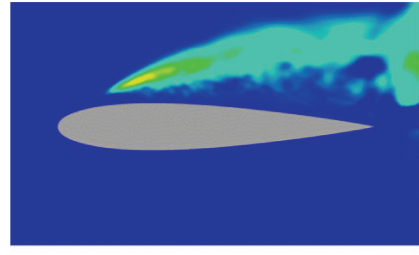

(a) DBD-off case

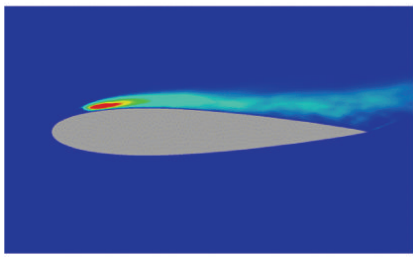

(d) Imt- 0.006 case

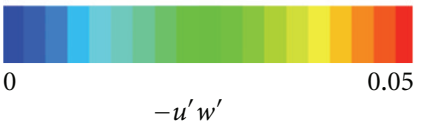

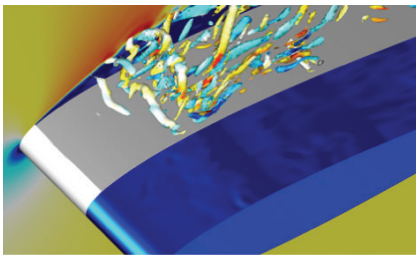

(a) DBD-off case

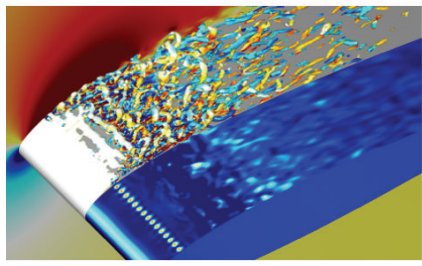

(d) Imt-0.006 case

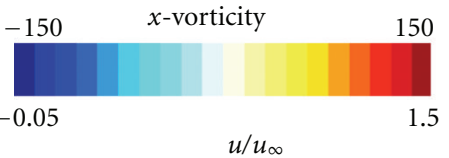

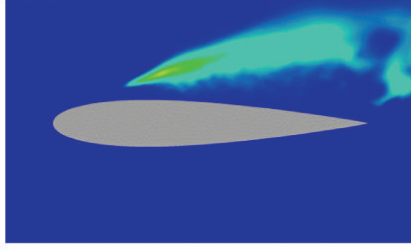

(b) Std-8 case

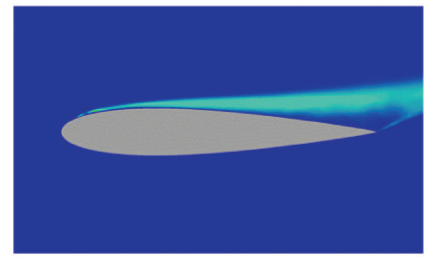

(e) Imt- 0.018 case

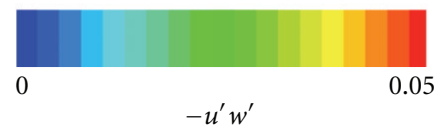

FIGURE 11: Reynolds stress distributions.

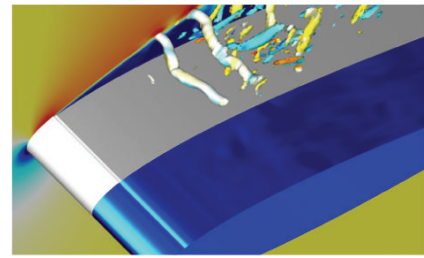

(b) Std-8 case

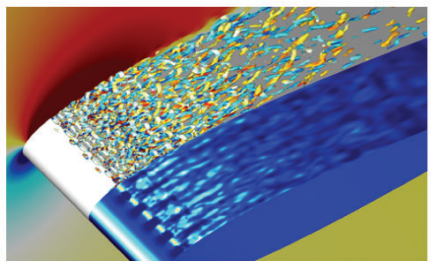

(e) Imt- 0.18 case $x$-vorticity

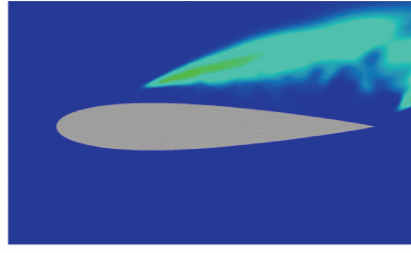

(c) Std-16 case

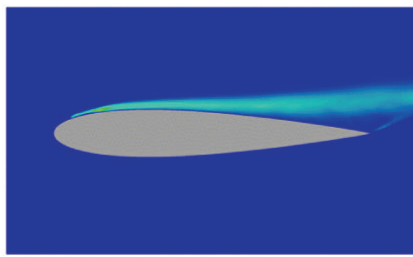

(f) Imt- 0.045 case

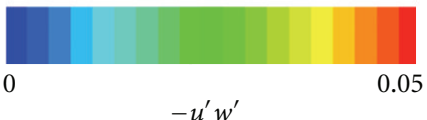

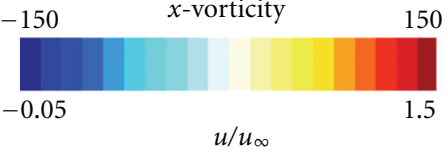

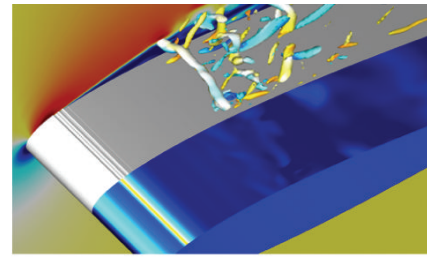

(c) Std-16 case

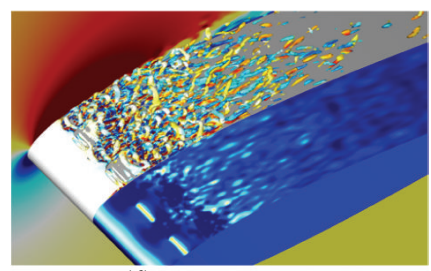

(f) Imt- 0.045 case

$-150 \quad x$-vorticity

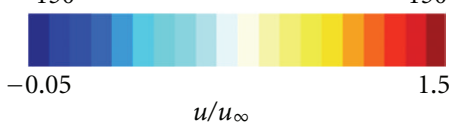

FIGURE 12: Instantaneous isosurfaces of the 2 nd invariant of velocity gradient tensors and chord direction velocity distributions. The value for the isosurface is 1250 .

the standard layout cases. As a result, very small threedimensional vortices are generated. It is considered that these vortices promote flow mixing and transfer the momentum from the freestream to the boundary layer.

In the Imt-0.018 case, as shown in Figure 14, twodimensional vortices are generated during the same period as the fluctuation in body force in the area where the body force is applied. In the area where the body force is not applied, three-dimensional vortices-such as hairpin vortices-are generated. In addition, small three-dimensional vortices are present between the older and the newly generated hairpin vortices. These hairpin and two-dimensional vortices break down rapidly during downstream transfer and change into smaller three-dimensional vortices. In a case where threedimensional vortices are generated closer to the leading edge, the separation is controlled more effectively. Thus, it is considered that the three-dimensional vortices behind the $5 \%$ chord length line from the leading edge contribute to flow mixing, and separation is controlled more effectively as a result.

In the Imt-0.045 case, as shown in Figure 15, the flow fields of vortices are similar to those in the Imt-0.018 case. 


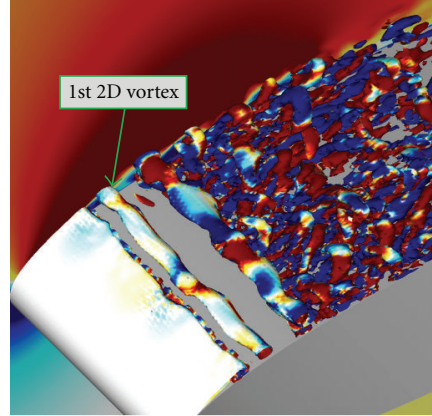

(a)

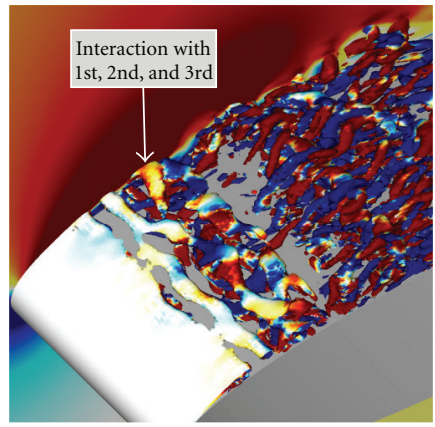

(d)

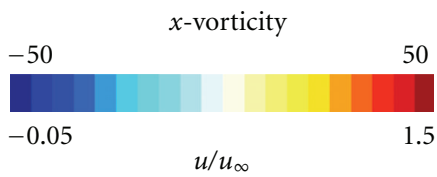

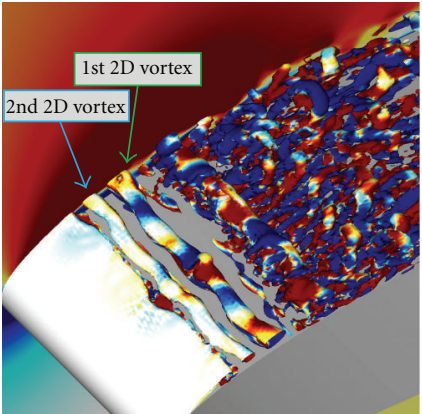

(b)

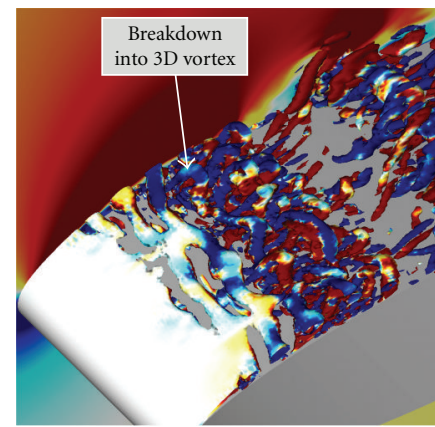

(e)

$x$-vorticity

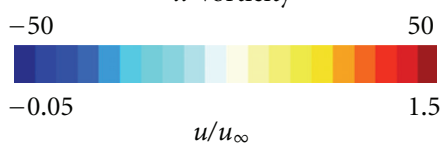

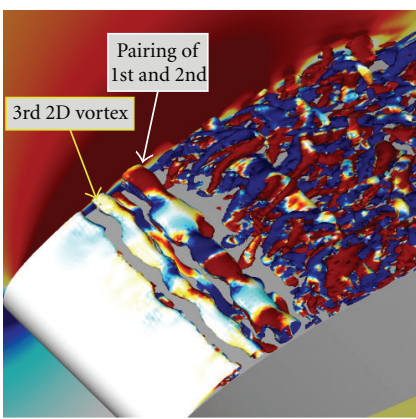

(c)

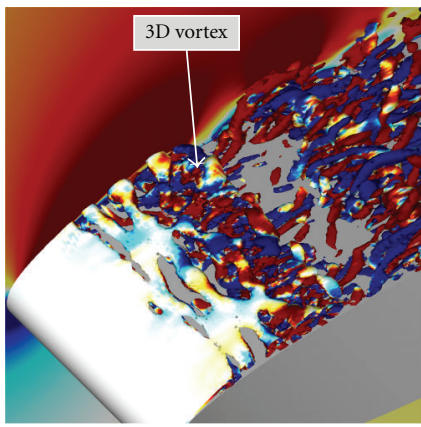

(f)

$x$-vorticity

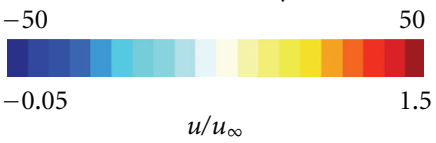

FIGURE 13: Time series visualization of isosurfaces of the 2nd invariant of velocity gradient tensors and chord direction velocity distributions (Imt- 0.006 case). The value for the isosurface is 1250 .

However, in this case, hairpin vortices-such as in the Imt0.018 case-do not exist. This factor probably leads to the effect of separation control in the Imt- 0.045 case being less effective than that in the Imt- 0.018 case. As mentioned above, it is important for separation control to generate threedimensional vortices in addition to the effect of the $D_{c}$ value, and the separation area decreases by generating these three-dimensional vortices closer to the leading edge. In order to generate three-dimensional vortices by distributing the body force intermittently, the intermittent distance is very important because three-dimensional vortices are not generated if the distance is too small.

As discussed above, the Imt -0.018 case is the most effective for controlling separated flows. It may be due to the generation of hairpin vortices, which is typically observed in this case as discussed previously. Therefore, the generation mechanism of these hairpin vortices is discussed briefly. The generation mechanism of hairpin vortices observed in the Imt-0018 case is different from that observed in the usual turbulent boundary layer.

Figure 16 is a schematic showing the generation process of hairpin vortices, and Figure 17 shows the flow field for each stage of the generation process. Here, spatial averaging is conducted for the flow fields by assuming the spatial periodicity of the flow fields. These averaged flow fields clearly show the existence of hairpin vortices. This illustrates that the generation of these hairpin vortices is not incidental but inevitable for this type of layout. The green regions in Figure 16 show the location of the body forces.

The first stage shown in Figures 16 and 17 illustrates that spanwise vortices are observed in the region where body force is not added. This is because the flow in the region where body force is added is attached and spanwise vortices are not generated there. The second stage shown in Figure 16 illustrates that the flow induced by the body force impinges into the body surface, and these flows blow into the region where the body force is not added. This flow is directed away from the airfoil surface. Thus, the spanwise vortices emitted from the separated shear layer are bent in the $\zeta$ direction. The flow impinges at the center of the region where the body force is not acted upon, and this flow continues in the direction along the airfoil surface. This flow induces longitudinal vortices, in which the axes are in the chord direction. Next, as shown in the third stage in Figure 16, the head of the spanwise vortices bent in the $\zeta$-direction moves in the $\xi$-direction and hairpin vortices are generated, as shown in Figure 17. The longitudinal vortices generated in the second stage exist at the downside of these hairpin vortices. Figures 16 and 17 show that these hairpin and longitudinal vortices are stretched toward the downstream, 


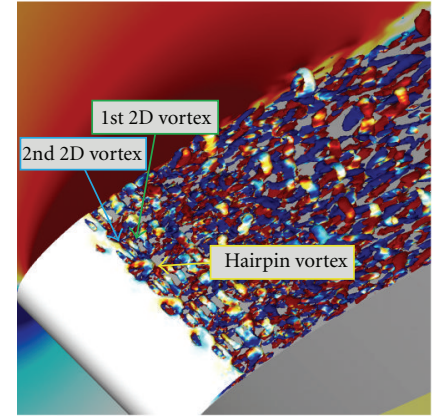

(a)

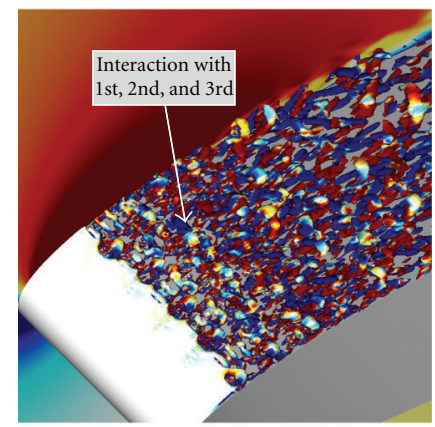

(d)

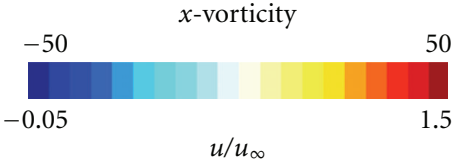

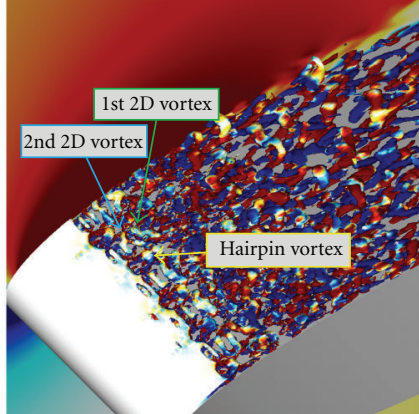

(b)

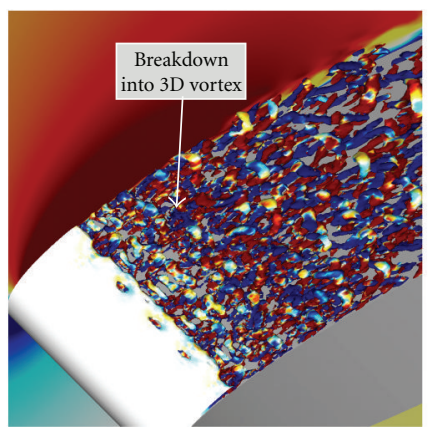

(e)

$x$-vorticity

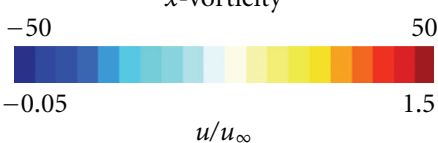

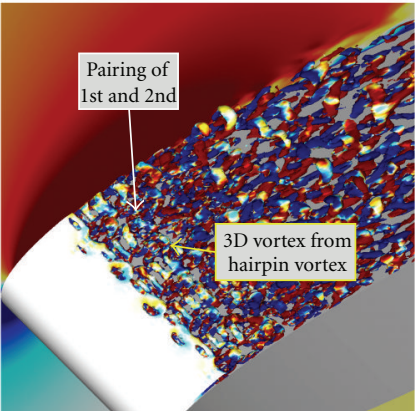

(c)

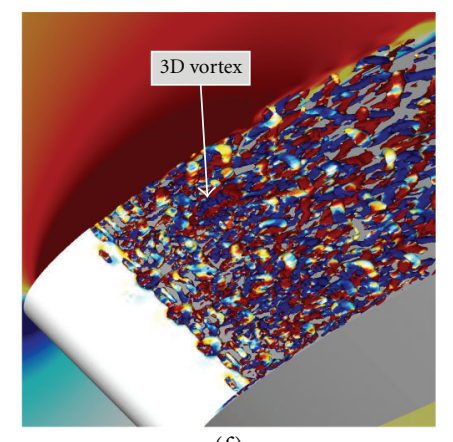

(f)

$x$-vorticity

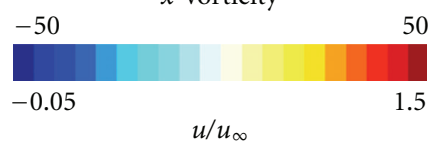

FIGURE 14: Time series visualization of isosurfaces of the 2nd invariant of velocity gradient tensors and chord direction velocity distributions (Imt-0.018 case). The value for the isosurface is 1250 .

and two pairs of longitudinal vortices, which have reversed vorticity to each other, are connected to the trailing edge.

In the Imt-0.018 case, hairpin vortices-as shown above-are periodically generated, and the effect of separation control is enhanced by the mixing of momentum due to the convection of these vortices. Interestingly, such clear hairpin vortices are only observed in the Imt- 0.018 case. In the Imt-0.006 case, longitudinal vortices are not generated because of a very small intermittent distance, whereas in the Imt-0.045 case, longitudinal vortices are not generated because of a very large intermittent distance. The hairpin vortices generated in the Imt- 0.018 case are similar to the turbulent boundary layer, although its generation process is very different. Therefore, the most stable intermittent distance should be similar to that in the turbulent boundary layer. In this study, the intermittent distance in the Imt0.018 case is approximately 100 in wall unit as shown in Table 1, which is similar to the streak width in the turbulent boundary layer. Here, the intermittent distance in wall unit in Table 1 is not exactly proportional to the actual distance because the wall velocity which is estimated from the shear stress changes with cases. On the other hand, the intermittent distances in the Imt- 0.006 and Imt-0.045 cases are very different from the streak width in the turbulent boundary layer. Hence, hairpin vortices are clearly observed only in the Imt- 0.018 case, which is the most effective case.

\section{Conclusions}

Two types of cases in which body force was distributed in a standard layout and an intermittent layout over an NACA 0015 airfoil were simulated by implicit large eddy simulations using a high-resolution compact scheme. The separated flow reattaches in the intermittent layout cases but does not do so in the standard layout cases. One of the probable reasons is that three-dimensional vortices promote flow mixing. In addition, in the intermittent layout cases, there is an optimum intermittent distance for separation control. This illustrates that the intermittent distance is important to the intentional generation of three-dimensional vortices. Our analysis shows that the optimum distance is comparable to the streak width in the turbulence boundary layer.

From this research, it is clear that generating threedimensional vortices by applying body force intermittently is effective in controlling the separation. In order to generate three-dimensional vortices intentionally, the intermittent distance should be specific.

\section{Nomenclatures}

a: Speed of sound

$c$ : Chord length

$c_{p}$ : Constant pressure-specific heat 


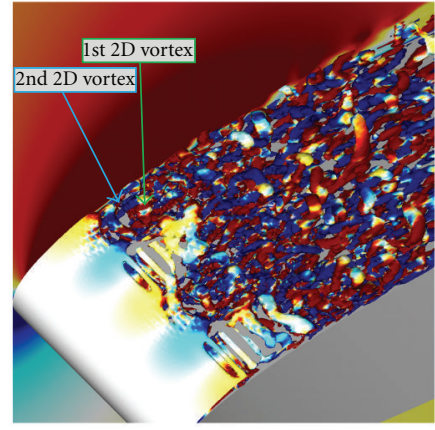

(a)

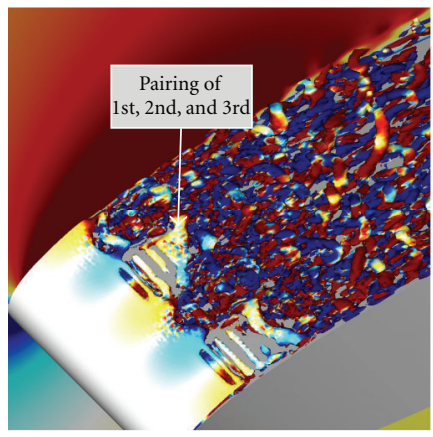

(d)

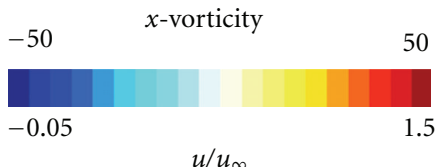

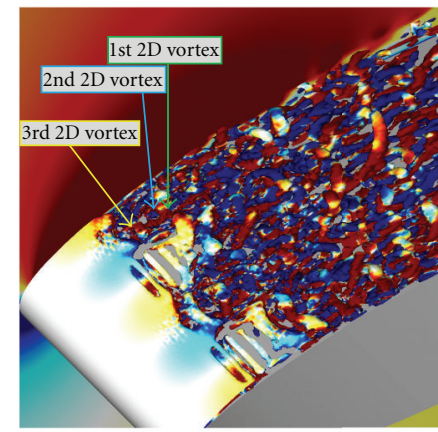

(b)

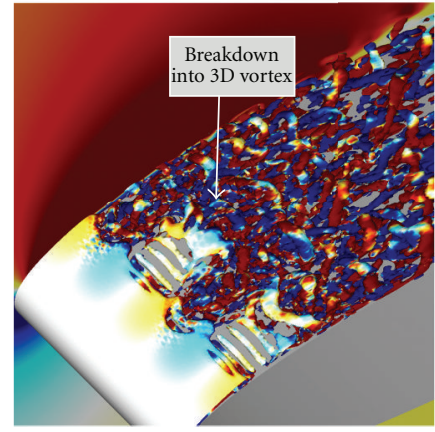

(e)

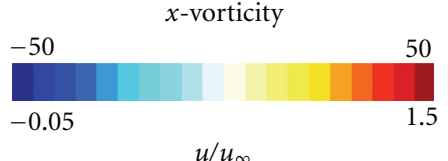

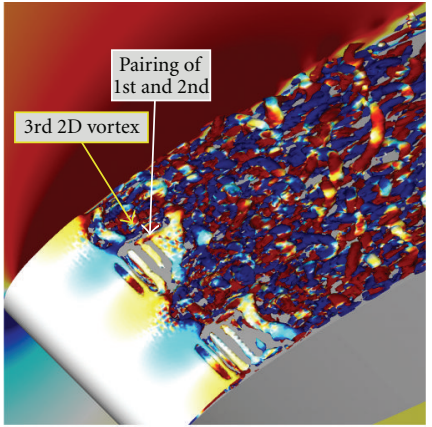

(c)

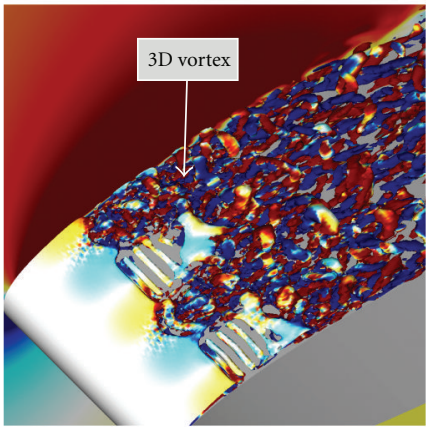

(f)

$x$-vorticity

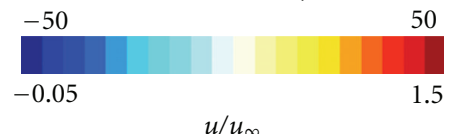

FIGURE 15: Time series visualization of isosurfaces of the 2 nd invariant of velocity gradient tensors and chord direction velocity distributions (Imt- 0.045 case). The value for the isosurface is 1250 .

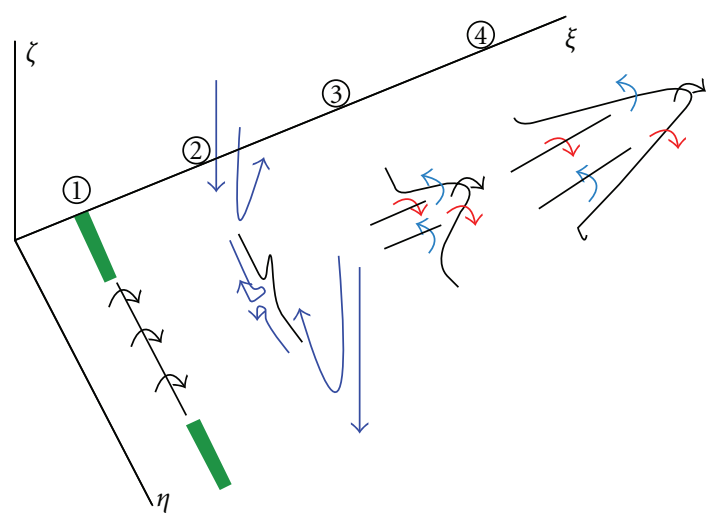

FIGURE 16: The process of generating a hairpin vortex.

$C_{f}:$ Friction coefficient

$C_{p}:$ Pressure coefficient

$D_{c}$ : Ratio between electrostatic body force added by plasma actuator and dynamic pressure

$D_{c \text {,ave }}$ : Spanwise averaged $D_{c}$. In standard case, $D_{c}=D_{c, \text { ave }}$. In intermittent case, $0.5 D_{c}=D_{c, \text { ave }}$

$e$ : $\quad$ Total energy per unit value
$E_{i}: \quad$ Electric field vector induced by plasma actuator

$f_{\text {base: }}$ Input voltage sine wave frequency

$F_{\text {base: }}$ Nondimensional base frequency for sine wave of input voltage, $F_{\text {base }}=f_{\text {base }} c / U_{\infty}$

$F^{+}$: Nondimensional burst frequency

$k$ : $\quad$ Heat conduction coefficient

$M_{\infty}$ : Freestream Mach number, $M_{\infty}=u_{\infty} / a_{\infty}$

p: $\quad$ Pressure

Pr: $\quad$ Prandtl number

$q_{i}: \quad$ Heat flux vector

$Q_{c}$ : $\quad$ Electric charge

Re: Reynolds number

$S_{i}: \quad$ Body force vector, $S_{i}=q_{c} E_{i}$

$S_{\text {suzen }}$ : Steady body force distribution determined from the Suzen model vector

$t$ : Time

T: $\quad$ Burst wave period

$T_{\text {on }}$ : Period when sine wave switch is "on" during burst wave period

$u$ : $\quad$ Chord direction velocity

$u^{\prime}$ : $\quad$ Fluctuation from Reynolds averaged $u$

$u^{\prime} w^{\prime}$ : Reynolds stress

$u_{\infty}$ : Freestream velocity

$u_{i}$ : Velocity vector

$V_{\text {ac }}$ : Voltage of alternator 


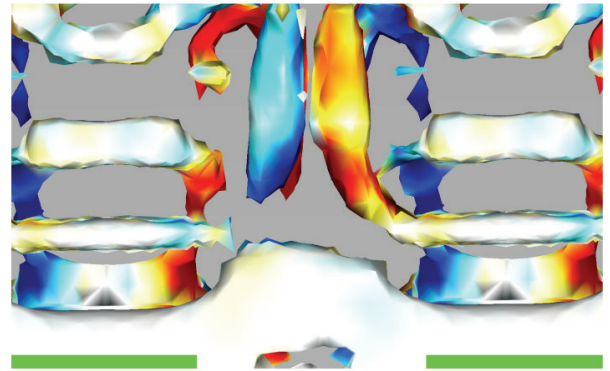

(a) First stage

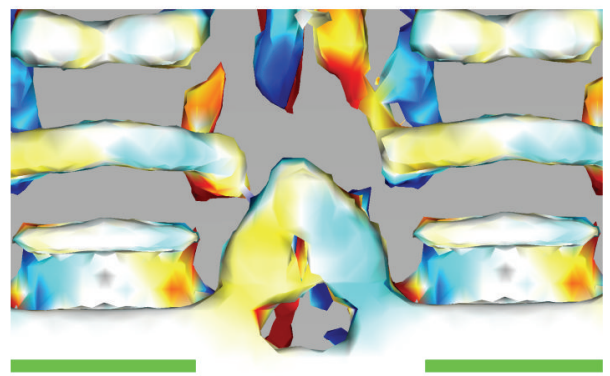

(c) Third stage $x$-vorticity

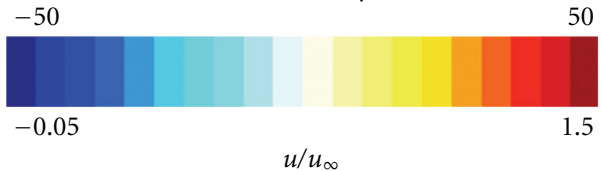

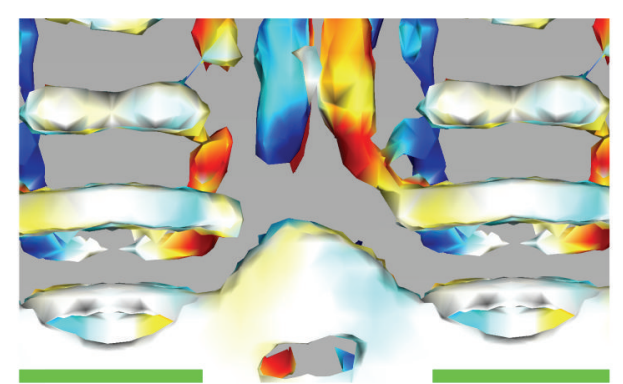

(b) Second stage

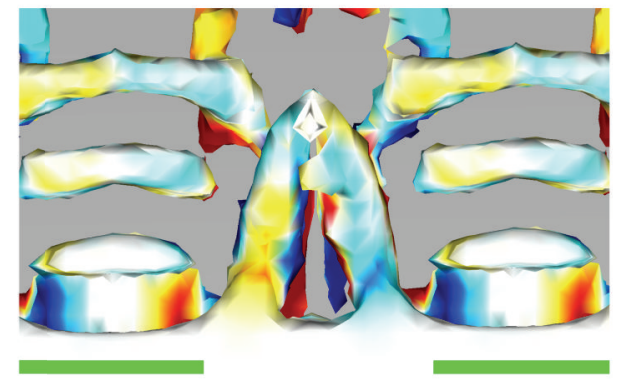

(d) Final stage $x$-vorticity

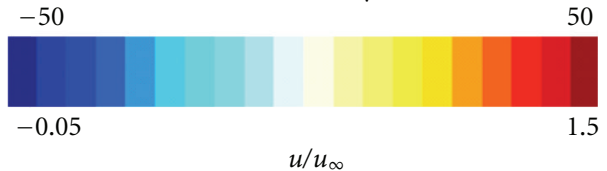

FIGURE 17: Top view of the hairpin vortex generated in the Imt-0018 case. The isosurfaces of the 2nd invariant of velocity gradient tensors colored by the $x$-direction vorticity are shown, where the value of the isosurface is 1250 .

w: $\quad$ Velocity in direction vertical to chord direction

$w^{\prime}: \quad$ Fluctuation from Reynolds averaged $w$

$x$ : Coordinate in the direction of chord length from the leading edge

$x_{i}$ : $\quad$ Position vector

$\alpha: \quad$ Angle of attack

$\delta_{i j}: \quad$ Kronecker delta

$\mu: \quad$ Viscosity

$\rho: \quad$ Density

$\tau_{i j}: \quad$ Viscose stress tensor

$\xi, \eta, \zeta:$ Body-fitted coordinate.

\section{Subscript}

ref: Reference value $\infty$ : Freestream condition.

\section{Acknowledgments}

The computational results are partially obtained through the research supported by a Grant-in-Aid for Scientific Research 20246122 in FY2010. The part of the postprocessing of the results is carried out through the research supported by Strategic Programs for Innovative Research (SPIRE) of High Performance Computing Initiative (HPCI) in FY2011.

\section{References}

[1] T. C. Corke, M. L. Post, and D. M. Orlov, "SDBD plasma enhanced aerodynamics: concepts, optimization and applications," Progress in Aerospace Sciences, vol. 43, no. 7-8, pp. 193217, 2007.

[2] M. P. Patel, T. T. Ng, S. Vasudevan, T. C. Corke, and C. He, "Plasma actuators for hingeless aerodynamic control of an unmanned air vehicle," Journal of Aircraft, vol. 44, no. 4, pp. 1264-1274, 2007.

[3] D. P. Rizzetta and M. R. Visbal, "Numerical investigation of plasma-based flow control for a transitional highlyloaded low-pressure turbine," in Proceedings of the 45th AIAA Aerospace Sciences Meeting, vol. 16, pp. 11366-11388, 2007, AIAA 2007-938.

[4] D. K. Van Ness, T. C. Corke, and S. C. Morris, "Tip clearance flow control in a linear turbine cascade using plasma actuation," in Proceedings of the 47th AIAA Aerospace Sciences Meeting including the New Horizons Forum and Aerospace Exposition, p. 300, January 2009, AIAA 2009-300.

[5] A. A. Sidorenko, B. Y. Zanin, B. V. Postnikov et al., "Pulsed discharge actuators for rectangular wing separation control," in Proceedings of the 45th AIAA Aerospace Sciences Meeting, pp. 11422-11432, January 2007, AIAA 2007-941.

[6] D. Tsubakino, Y. Tanaka, and K. Fujii, "Effective layout of plasma actuators for a flow separation control on a wing," in Proceedings of the 45th AIAA Aerospace Sciences Meeting, vol. 9, pp. 5695-5704, 2007, AIAA 2007-474. 
[7] B. Goksel, D. Greenblatt, I. Rechenberg, C. N. Nayeri, and C. O. Paschereit, "Steady and unsteady plasma wall jets for separation and circulation control," in Proceedings of the $3 r$ d AIAA Flow Control Conference, pp. 1621-1635, San Francisco, Calif, USA, June 2006, AIAA 2006-3686.

[8] M. P. Patel, T. T. Ng, S. Vasudevan et al., "Scaling effects of an aerodynamic plasma actuator," in Proceedings of the 45th AIAA Aerospace Sciences Meeting, vol. 11, pp. 7654-7679, 2007, AIAA 2007-635.

[9] K. Asada and K. Fujii, "Computational analysis of unsteady flow-field induced by plasma actuator in burst mode," in Proceedings of The 5th Flow Control Conference, Chicago, Ill, USA, June 2010, AIAA 2010-5090.

[10] J. Poggie, C. P. Tilmann, P. M. Flick et al., "Closed-loop stall control on a morphing airfoil using hot-film sensors and DBD actuators," in Proceedings of The 48th AIAA Aerospace Sciences Meeting, Orlando, Fla, USA, January 2010, AIAA 2010-547.

[11] D. P. Rizzetta and M. R. Visbal, "Numerical investigation of plasma-based control for low-reynolds number airfoil flows," in Proceedings of the 5th Flow Control Conference, June 2010, AIAA 2010-4255.

[12] K. Asada, Y. Ninomiya, A. Oyama, and K. Fujii, "Airfoil flow experiment on the duty cycle of DBD plasma actuator," in Proceedings of The 47th AIAA Aerospace Sciences Meeting including the New Horizons Forum and Aerospace Exposition, Orlando, Fla, USA, January 2009, AIAA 2009-0531.

[13] Y. B. Suzen and P. G. Huang, "Simulations of flow separation control using plasma actuators," in Proceedings of The 44th AIAA Aerospace Sciences Meeting, pp. 10456-10464, January 2006, AIAA 2006-877.

[14] C. L. Enloe, M. G. McHarg, G. I. Font, and T. E. McLaughlin, "Plasma-induced force and self-induced drag in the dielectric barrier discharge aerodynamic plasma actuator," in Proceedings of The 47th AIAA Aerospace Sciences Meeting including the New Horizons Forum and Aerospace Exposition, Orlando, Fla, USA, January 2009, AIAA 2009-1622.

[15] K. Fujii, "Developing an accurate and efficient method for compressible flow simulations-example of CFD in aeronautics," in Proceedings of the 5th International Conference on Numerical Ship Hydrodynamics, 1990.

[16] S. Obayashi, K. Matsushima, K. Fujii, and K. Kuwahara, "Improvements in efficiency and reliability for navier-stokes computations using the LU-ADI factorization algorithm," in Proceedings of the 24th AIAA Aerospace Sciences Meeting, New York, NY, USA, 1986, AIAA 86-0513.

[17] S. K. Lele, "Compact finite difference schemes with spectrallike resolution," Journal of Computational Physics, vol. 103, no. 1, pp. 16-42, 1992.

[18] M. R. Visbal and D. V. Gaitonde, "Computation of aeroacoustic fields on general geometries using compact differencing and filtering schemes," in Proceedings of The American Institute of Aeronautics and Astronautics, 1999, AIAA 1999-3706.

[19] D. V. Gaitonde and M. R. Visbal, "Pade-type higher-order boundary filters for the navier-stokes equations," AIAA Journal, vol. 38, pp. 2103-2112, 2000.

[20] H. Nishida and T. Nonomura, "ADI-SGS scheme on ideal magnetohydrodynamics," Journal of Computational Physics, vol. 228, no. 9, pp. 3182-3188, 2009.

[21] M. R. Visbal and D. P. Rizzetta, "Large-eddy simulation on general geometries using compact differencing and filtering schemes," in Proceedings of The American Institute of Aeronautics and Astronautics, 2002, AIAA 2002-288.
[22] K. Fujii, "Unified zonal method based on the fortified solution algorithm," Journal of Computational Physics, vol. 118, no. 1, pp. 92-108, 1995. 

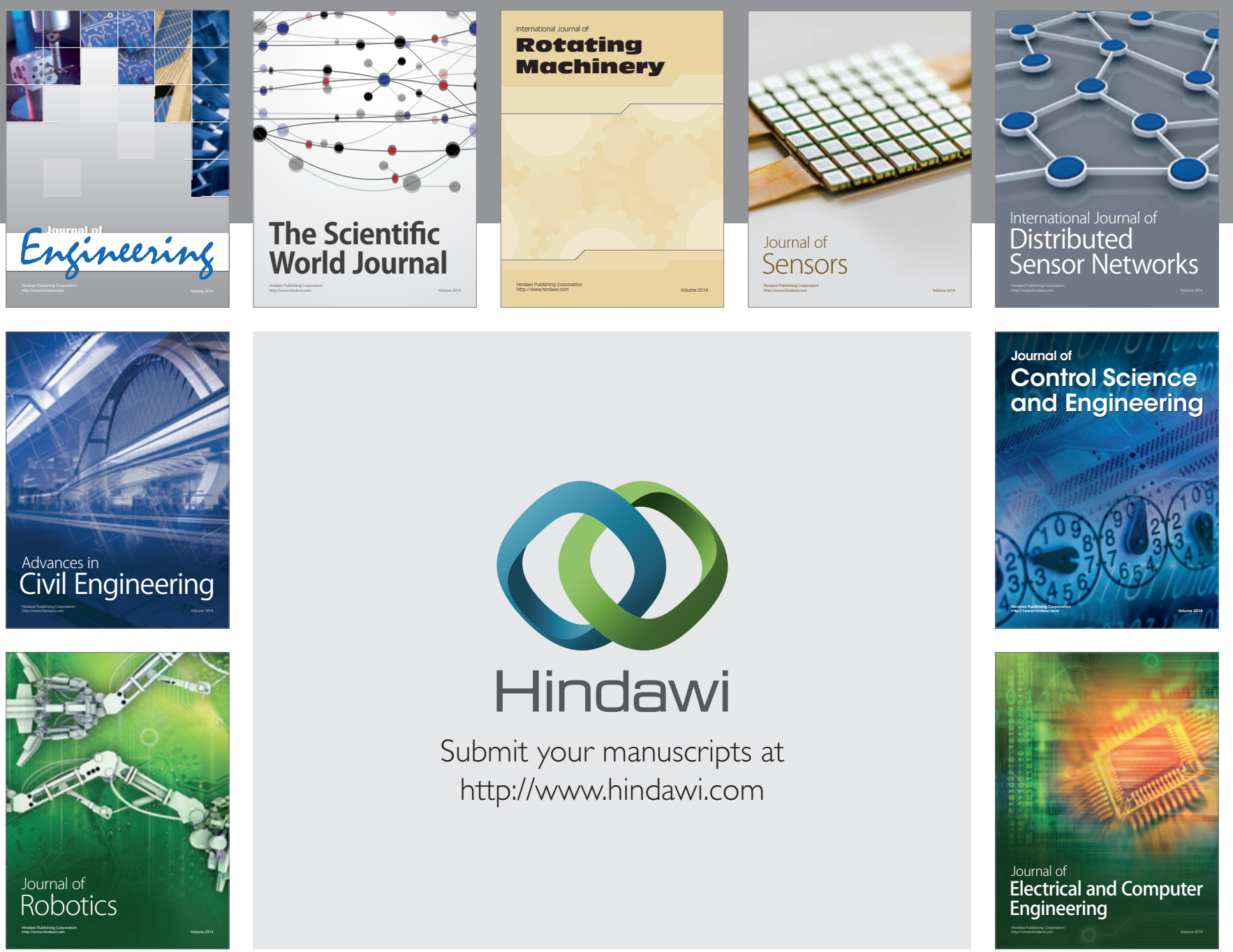

Submit your manuscripts at

http://www.hindawi.com
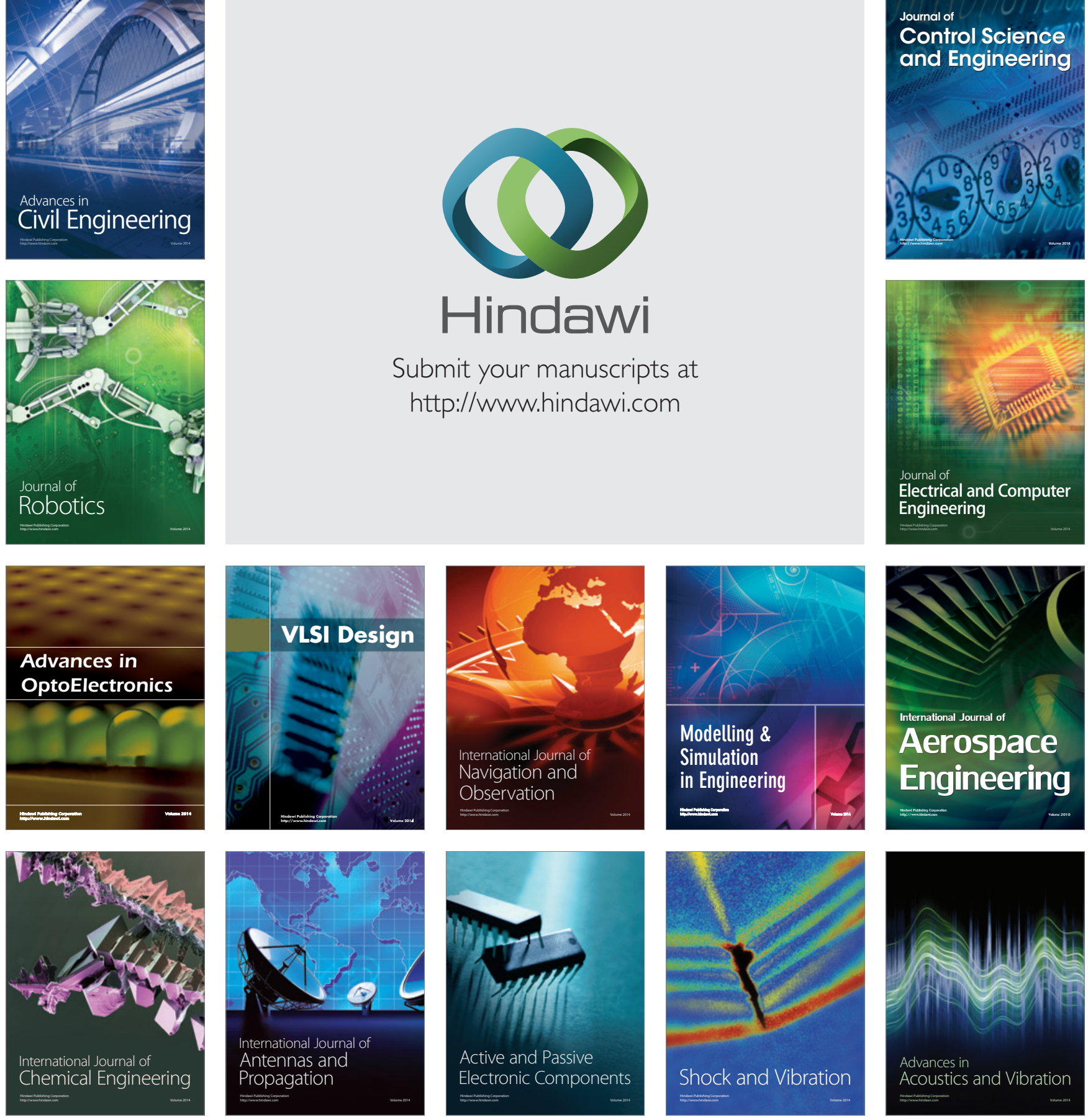